\title{
Neuromedin $\mathrm{U}$ receptor 2 knockdown in the paraventricular nucleus modifies behavioral responses to obesogenic high-fat food and leads to increased body weight
}

\author{
Caitlin R. Benzon, Sarah B. Johnson, David L. McCue, Dingge Li, Thomas A. Green, and \\ Jonathan D. Hommel \\ Center for Addiction Research, Department of Pharmacology \& Toxicology, University of Texas \\ Medical Branch
}

\section{Abstract}

Neuromedin $\mathrm{U}(\mathrm{NMU})$ is a highly conserved neuropeptide which regulates food intake and body weight. Transgenic mice lacking NMU are hyperphagic and obese, making NMU a novel target for understanding and treating obesity. Neuromedin U receptor 2 (NMUR2) is a high-affinity receptor for NMU found in discrete regions of the central nervous system, in particular the paraventricular nucleus of the hypothalamus (PVN), where it may be responsible for mediating the anorectic effects of NMU. We hypothesized that selective knock down of NMUR2 in the PVN of rats would increase their sensitivity to the reinforcing properties of food resulting in increased intake and preference for high-fat obesogenic food. To this end, we used viral-mediated RNAi to selectively knock down NMUR2 gene expression in the PVN. In rats fed a standard chow, NMUR2 knockdown produced no significant effect on food intake or body weight. However, when the same rats were fed a high-fat diet ( $45 \%$ fat), they consumed significantly more food, gained more body weight, and had increased feed efficiency relative to controls. Furthermore, NMUR2 knockdown rats demonstrated significantly greater binge-type food consumption of the high-fat diet and showed a greater preference for higher-fat food. These results demonstrate that NMUR2 signaling in the PVN regulates consumption and preference for high-fat foods without disrupting feeding behavior associated with non-obesogenic standard chow.

\section{Keywords}

Obesity; Dietary fat; Neuromedin U receptor 2; Feeding behavior; Viral RNA interference; Binge eating

\begin{abstract}
Over 500 million people, or $10 \%$ of the worldwide population, are obese and this number continues to rise at an alarming rate (Finucane et al., 2011). In the United States, the rate is even higher with $35.7 \%$ of the adult population being obese (Ogden et al., 2012). Numerous comorbidities are associated with obesity including diabetes, heart disease, dyslipidemia, and cancer (Guh et al., 2009), placing a large burden on healthcare systems. Unfortunately, the available treatment options either require invasive surgery (e.g. bariatric surgery) or have
\end{abstract}

(C) 2013 IBRO. Published by Elsevier Ltd. All rights reserved.

Corresponding author: Jonathan D. Hommel, University of Texas Medical Branch, 301 University Blvd, Galveston, TX 77555-0615, jdhommel@utmb.edu, Office Phone: 409-747-7057.

Publisher's Disclaimer: This is a PDF file of an unedited manuscript that has been accepted for publication. As a service to our customers we are providing this early version of the manuscript. The manuscript will undergo copyediting, typesetting, and review of the resulting proof before it is published in its final citable form. Please note that during the production process errors may be discovered which could affect the content, and all legal disclaimers that apply to the journal pertain. 
modest efficacy (e.g., Orlistat, diet and exercise). At the root of this problem is overconsumption of foods which are high in fat and sugar. These energy dense foods are widely considered to be obesogenic (Warwick and Schiffman, 1992, West and York, 1998). Behavioral and molecular mechanisms which specifically regulate consumption of obesogenic foods are poorly understood. Therefore, an expanded appreciation of the molecular substrates which regulate consumption of obesogenic food is imperative.

A candidate in this regard is neuromedin $\mathrm{U}$ (NMU), a highly conserved neuropeptide shown to regulate food intake (Howard et al., 2000) and body weight (Hanada et al., 2004). NMU has two high affinity receptors: Neuromedin $U$ receptor 1 , which is primarily expressed in the periphery, and NMUR2 which is primarily expressed in the central nervous system (Brighton et al., 2004). However, the phenotypes of two separate NMUR2 knockout mouse lines do not agree. Egecioglu et al report that NMUR2 knockout mice fed a high-fat diet have increased body weight (Egecioglu et al., 2009). It is unclear if this effect is a consequence of developmental alterations, metabolic changes, or modified food intake. Furthermore, Peier et al report that NMUR2 knockouts have decreased body weight and adiposity, regardless of the type of diet (Peier et al., 2009). These discrepancies cloud our understanding of NMUR2 function in vivo, and suggest that more refined studies of NMUR2 function are necessary.

Here, we present the first behavioral data on conditional knock down of NMUR2 in vivo. We employed adeno-associated virus (AAV) mediated RNAi to knock down NMUR2. The AAV was engineered to express either a small hairpin RNA (shRNA) designed to knock down NMUR2 (shNMUR2) or a control non-silencing hairpin (shCTRL). We administered AAV-shNMUR2 or AAV-shCTRL virus directly to the PVN of adult rats resulting in NMUR2 knockdown (shNMUR2 ${ }^{\mathrm{PVN}}$ ) and control (shCTRL ${ }^{\mathrm{PVN}}$ ) animals, respectively. Viral RNAi provides regional specificity for the knockdown based on the location of the virus injection, allowing more precise interpretation of the importance of specific brain regions. Furthermore, this technique avoids developmental confounds by providing temporal control over the knockdown based on the age of the animal when the AAV is injected. The role of NMUR2 in the PVN on feeding behaviors such as food preference and binge-type eating is unknown. Therefore, we assessed feeding behavior in rats with viral RNAi mediated knock down of NMUR2 in the PVN.

\section{Experimental Procedures}

\subsection{Subjects}

Twenty-four male Sprague-Dawley rats (Harlan, Inc.) weighing 225-250 grams at the start of the experiments were used. Rats were single-housed at $71^{\circ} \mathrm{F}$ and $30-50 \%$ relative humidity with a 12 hour light-dark cycle (lights on 6:00 am-6:00 pm) and were allowed to habituate for 7 days prior to any experimentation. All experiments were carried out in accordance with the Guide for the Care and Use of Laboratory Animals (Institute of Laboratory Animal Resources (U.S.), 1996) and with the approval of the Institutional Animal Care and Use Committee at The University of Texas Medical Branch. For behavioral studies, 12 animals received injections of AAV-shNMUR2 and 12 animals received AAV-shCTRL. After evaluating perfused brain slices containing each animal's PVN (including the bilateral injection sites, and GFP-labeled viral transduction) under a microscope, only animals with bilateral AAV transduction were used for data analysis. Based on this bilateral transduction criterion, we excluded three animals from the AAVshNMUR2 group leaving $n=9$, and five animals from the AAV-shCTRL group leaving $n=7$. 


\subsection{Hairpin design}

Hairpin RNA was designed to target NMUR2 rat mRNA. Ten different 24-nucleotide sequences, oriented $5^{\prime}$ to $3^{\prime}$, were identified within the mRNA sequence (Ensembl transcript ID ENSRNOT00000018967) using search algorithms from previously described criteria (Hommel et al., 2003). The 24-nucleotide sequences also needed to be relatively unique within the rat genome to minimize off-target effects of RNAi. The top strand of the hairpin oligonucleotide was constructed starting $5^{\prime}$ with the antisense of the 24-nucleotide sequence joined to the sense sequence by a 10-nucleotide miRNA loop (5'-CTTCCTGTCA-3'; Kawasaki, H. \& Taira, K., 2003). The final oligonucleotide sequences were synthesized (Sigma-Aldrich) as follows: shNMUR2-5 top 5'-

TTGAACACAGCAGCCAGGGACTCTGTCTTCCTGTCAACAGAGTCCCTGGCTGCT GTGTTATTTTT- $3^{\prime}$ and shNMUR2-5 bottom $5^{\prime}$ CTAGAAAAATAACACAGCAGCCAGGGACTCTGTTGACAGGAAGACAGAGTCCC TGGCTGCTGTGTTC- $3^{\prime}$.

A pAAV-shRNA and a plasmid already containing the control hairpin, shCTRL were donated by Ralph DiLeone and contained the following insert: top 5'-

TTTGTGGAGCCGAGTTTCTAAATTCCGCACCGGAATTTAGAAACCCGGCTCCAC TTTTT-3'; bottom 5'-

CTAGAAAAAGTGGAGCCGGGTTTCTAAATTCCGGTGCGGAATTTAGAAACTCGG CTCCA-3'. The hairpin vector was designed to co-express enhanced green fluorescent protein (GFP) under the control of an independent RNA Polymerase II promoter and terminator. Annealed hairpin oligonucleotides were cloned into the pAAV-shRNA plasmid. RNAi viruses were produced using a triple-transfection, helper-free method and purified as previously described (Hommel et al., 2003).

\subsection{In vitro hairpin screening}

Ten pAAV-shNMUR2 hairpin vectors and one pAAV-shCTRL vector were tested for knockdown efficacy in vitro. To create an NMUR2 expression vector, the rat NMUR2 gene sequence (NCBI NM022275.2) was synthesized (GenScript), then cloned into the pAAVMCS expression plasmid (Stratagene). For hairpin screening, HEK-293 cells were transfected using a standard Lipofectamine 2000 protocol (Invitrogen). For each hairpin, cells were double-transfected with the NMUR2 expression vector and the hairpin vector at a gene vector-to-hairpin ratio of 1 to 10. Similarly, shCTRL was double-transfected along with the same proportion of the gene expression vector. Two days post-transfection, RNA was extracted (RNeasy Mini kit, Qiagen), treated with RNase-free DNase (Qiagen), and synthesized into cDNA (iScript cDNA Synthesis kit, Bio-Rad). Knockdown efficacy for each hairpin and for shCTRL was measured with Real-Time PCR (7500 Fast Real Time PCR system, Applied Biosystems), which quantified differences in NMUR2 mRNA as $\triangle \Delta \mathrm{CT}$, using GAPDH as the normalizing control.

To screen for potential off-target knockdown effects of shNMUR2, we performed a similar Real-Time PCR analysis of in vitro mRNA expression. We selected 6 rat genes that contained the closest matches to the 24-nucleotide sequence of the best shNMUR2 hairpin (shNMUR2 \#5, identified in the previous experiment). The most similar sequences found in the rat genome matched 16 out of 24 nucleotides. We designed primers to target each gene and had the oligonucleotides synthesized (Integrated DNA Technologies, Inc.) as follows: Abcg311 Forward 5'-TCCTGATCCTCCTGCTCACT-3'; Abcg311 Reverse 5'CGATCCTGGTCAATCACAAA-3'; Mcm5 Forward 5'GCCAAGGAGGTAGCTGATG-3'; Mcm5 Reverse 5'GGCATCTGACTTGAGCATGA-3'; Mnat1 Forward 5'GTGTGGCACTCCACTGAGAA-3'; Mnat1 Reverse 5'- 
CCGAATTTCAACCTCCTTGT-3'; Rabl3 Forward 5'-

GGGACCCCTGAAGAGAAGAC-3'; Rabl3 Reverse 5'-

CGCTCTTGTGCTTTTCACAC-3'; RGD1309492 Forward 5'-

AGGACTCCGGCTTTTCAGAT-3'; RGD1309492 Reverse 5'-

GCTCACCACGTCCTCTGACT-3'; Slc5a7 Forward 5'-

CTGGGTTGGAGGAGGTTACA-3'; Slc5a7 Reverse 5'-

GAGCCCAAGCTAGACCACAA- $3^{\prime}$. In this experiment, we used PC-12 cells since they are derived from rat tissue, instead of HEK293 cells derived from humans, to more accurately predict potential off-target effects in vivo. We followed the same double-transfection, RNA extraction, purification, and cDNA synthesis protocols as in the in vitro hairpin screening experiment, except we only co-transfected the NMUR2 expression vector with the shNMUR2 \#5 knockdown hairpin, as well as shCTRL for controls. Using Real-Time PCR, we quantified mRNA levels of NMUR2 and the 6 selected genes in samples transfected with shNMUR2 or shCTRL. $\mathrm{C}_{\mathrm{T}}$ values were first normalized to GAPDH and 18S mRNA expression, quantified for each sample. Relative differences in target mRNA expression between treatments were quantified as $\Delta \Delta \mathrm{CT}$ then converted into fold change and percent knockdown.

\subsection{Microinjection surgery}

After arriving, rats were allowed to acclimate for 1 week before surgery. At time of surgery, rats were approximately 60 days old. Rats were maintained under anesthesia with an isoflurane vaporizer system (VetEquip) throughout the procedure. Anesthetized rats were shaved and secured on a stereotaxic apparatus (Kopf), a small scalp incision was made down the midline to expose the skull, and bilateral holes were drilled through the skull above the injection sites. Coordinates for the PVN injection site were adjusted for a needle to enter the brain at a $10^{\circ}$ outside angle, and were set at $1.8 \mathrm{~mm}$ posterior to bregma, $1.5 \mathrm{~mm}$ lateral from the midline, and $8.2 \mathrm{~mm}$ ventral to the surface of the skull (Paxinos and Watson, 2005). Rats were microinjected with $2 \mu \mathrm{L}$ of AAV into the PVN on each side at a rate of $0.2 \mu \mathrm{L}$ every 30 seconds for 5 minutes. Twelve rats received AAV-shNMUR2 and twelve rats received AAV-shCTRL. Post-injection, the needles remained in place for 5 minutes and then were removed. The incision was stapled and dressed with lidocaine and triple antibiotic ointment, and $0.2 \mathrm{~mL}$ bupivicaine was subcutaneously injected lateral to the incision. Rats were given an intra-muscular injection of $0.1 \mathrm{~mL}$ penicillin, placed in a clean cage on a heating pad, and allowed to recover from anesthesia under observation before being returned to the colony room. Rats were allowed to recover for at least 2 weeks post-surgery before starting behavioral experiments.

\subsection{Immunohistochemistry}

Following behavioral experimentation (90 days post viral injection), rats were anesthetized with a combination of ketamine and xylazine and transcardially perfused for 5 minutes with 1XPBS $(75 \mathrm{~mL})$, followed by 15 minutes of $4 \%$ paraformaldehyde in 1XPBS $(225 \mathrm{~mL})$. Brains were post-fixed overnight in $4 \%$ paraformaldehyde in 1XPBS, then cryoprotected with $20 \%$ glycerol plus $0.01 \%$ sodium azide in 1XPBS for at least 24 hours. Brains were then frozen with dry ice; using a microtome (Leica SM 2010R), 40 $\mu$ m coronal sections containing the PVN were collected and stored in vials of $0.01 \%$ sodium azide in 1 XPBS at $4^{\circ} \mathrm{C}$ until use. Sections were mounted onto Superfrost Plus microscope slides and allowed to dry for 2 hours. Slides were rehydrated in 1XPBS for 20 minutes, followed by ethanol dehydration and two 3-minute Citrisolve washes, and then were cover-slipped with DPX mounting medium. Targeting for each rat was assessed by examining native GFP expression under a fluorescent microscope (BX51, Olympus, Melville, NY). 
After assessment of viral expression and targeting, brains from five shCTRL and five shNmUR2-5 rats were chosen from the study group for further visual analysis through immunofluorescent double labeling with rabbit polyclonal anti-NMUR2 antibody (Novus Biologicals) and chicken anti-GFP antibody (Aves Labs) as primary antibodies. Sections were incubated for 5 minutes in 1\% SDS for antigen unmasking, washed 3 times in 1XPBS for 5 minutes, then incubated for 1 hour in a blocking solution containing: 3\% normal goat serum, $3 \%$ normal donkey serum, and $0.3 \%$ Triton X-100 in 1XPBS. Primary antibodies were diluted in blocking solution (rabbit anti-NMUR2, $2.5 \mu \mathrm{g} / \mathrm{mL}$; chicken anti-GFP, 20 $\mu \mathrm{g}$ / $\mathrm{mL}$ ). Sections were incubated in primary antibody solutions overnight (20 hours) at room temperature. Fluorescent secondary antibodies, Alexa-Fluor 568 donkey anti-rabbit (Invitrogen) and Cy2-conjugated goat anti-chicken (Jackson ImmunoResearch) were both diluted in 1XPBS at 1:100. Sections were washed 3 times in 1XPBS for 5 minutes, before incubating in the secondary antibody solution for 2 hours at room temperature. Sections were washed 3 times in 1XPBS for 5 minutes before being counterstained with DAPI (Invitrogen), mounted, and cover slipped with Vectashield Hard-Set Mounting Medium (Vector Laboratories). Figure 1 shows labeled sections of PVN adjacent to viral injection sites (injection sites not shown). Sections were imaged as z-stacks on a Leica DMI4000 Inverted TCS SPE confocal microscope. The images in Figure 1 represent single optical slices from the collected z-stacks and were selected for comparable viral expression pattern and similar medial location within the PVN's anterior-posterior plane. The composite images were processed using ImageJ. To highlight co-localization, we selected three neurons in shCTRL images to mark with white arrows.

\subsection{In vitro antibody validation}

To confirm primary and secondary antibody specificity for labeling NMUR2-containing cells, in vitro antibody validation was performed using immunofluorescent techniques. CHO-K1 cells were cultured in 4 chambers on Lab-Tek II chamber slides (Nunc). Three chambers were transfected with NMUR2 expression vector using a standard Lipofectamine 2000 protocol. One chamber was excluded as a non-transfected control.

Immunocytochemistry began two days post-transfection. Washes and incubations were done at room temperature unless noted. Cells were given two 3-minute washes in DPBS then fixed for 20 minutes in 4\% paraformaldehyde in PBS, $\mathrm{pH} 7.4$, followed by 3 more 3-minute washes. After fixation, cells were permeabilized for 10 minutes in $0.3 \%$ Triton X-100 in PBS, and washed again 3 times for 3 minutes. Cells were incubated for 1 hour in a blocking buffer of 5\% normal donkey serum and $0.1 \%$ Triton X-100 in PBS, then washed with DPBS 3 times for 5 minutes each. Primary antibody, polyclonal rabbit anti-NMUR2, was diluted in blocking buffer (1:150, final concentration $2 \mu \mathrm{g} / \mathrm{mL})$. Cells were incubated with primary antibody overnight at $4^{\circ} \mathrm{C}$, except for the $1^{\circ}$ antibody control, which received only blocking buffer. In the morning, cells were washed in DPBS 3 times for 5 minutes before secondary antibody incubation. Secondary fluorescent antibody, donkey anti-rabbit Alexa-Fluor 568, was diluted in DPBS (1:100). Cells were incubated with secondary antibody for 1 hour, except for secondary antibody control, which received only DPBS. After secondary antibody incubation, cells were given three 5-minute washes in DPBS before the plastic chambers were detached from the slides. Slides were cover-slipped with Vectashield Hard-Set Mounting Medium with DAPI (Vector Laboratories) and stored at $4{ }^{\circ} \mathrm{C}$ overnight to harden. Primary antibody specificity was validated through visual analysis using a fluorescent microscope (BX51, Olympus, Melville, NY) and cells were imaged with a high-resolution digital microscope camera (Hamamatsu C4742-95, Hamamatsu Photonics, Hamamatsu City, Japan). 


\subsection{Food intake and body weight}

Food intake and body weights were monitored daily for 2 weeks, then at least twice a week for 3 months, using a dynamic weighing apparatus (Ohaus Corp). All measurements were taken in the animal holding room between 2:00 pm and 3:00 pm. Rats were maintained on a standard chow (Teklad Mouse/Rat Diet 7912, Harlan) unless otherwise noted. Standard chow contains $17 \% \mathrm{kcal}$ from fat, $58 \%$ from carbohydrate, $25 \%$ from protein, and provides energy of $3.1 \mathrm{kcal} / \mathrm{gm}$ (Table 1). High-fat diet used for high fat food intake and binge-type eating studies contains $45 \% \mathrm{kcal}$ from fat, $35 \%$ carbohydrate, $20 \%$ protein, and provides 4.73kcal/gm (Open Source Diets formula D12451, Research Diets Inc., New Brunswick, NJ, USA).

\subsection{Sucrose preference \& Locomotor Activity}

Sucrose preference and locomotor activity were performed as previously described (Hommel et al., 2006). In short, both bottles were initially filled with reverse osmosis (RO) water to establish a baseline preference over two days. On day three, bottles were weighed, emptied, and one bottle was filled with RO water, while the other bottle was filled with a $0.25 \%$ sucrose solution in RO water. Bottle weights were recorded and placed back into the rat cages. Day 4, bottles were re-weighed, positions switched and placed back into the cages. This protocol was followed until we had tested the $0.25 \%, 0.50 \%$, and $1.00 \%$ sucrose solutions. All bottles were washed and randomized before increasing sucrose concentrations.

Novel locomotor activity was monitored and quantified using an open field activity system (San Diego Instruments, San Diego, CA, USA). Rats were acclimated to the locomotor activity room for three hours on the two separate days prior to test day. On test day, the rat cages were placed in a $4 \times 4$ matrix of photobeams which measured horizontal beam breaks, and a row of 16 photobeams which measured vertical (rearing) activity. Activity was measured over a 23 hour period.

\subsection{Binge-type eating behavior}

Our binge-type eating protocol is a limited-access model based on previous publications (Corwin, 2006, Corwin et al., 2011). Rats were switched from a standard chow to a $45 \%$ high-fat diet (Research Diets Inc., New Brunswick, NJ) for 1 week to minimize food neophobia. After 1 week, the diets were switched from $45 \%$ high fat diet back to standard chow for 1 week before testing. The day before the binge, normal intake of standard chow was recorded at the beginning of the dark cycle for 2 hours (from 6:00-8:00 pm). On the day of the binge, rats were given access to pre-measured amounts of $45 \%$ high-fat diet at the beginning of the dark cycle for 2 hours. At the end of 2 hours, total intake of the $45 \%$ highfat diet was recorded. Animals were returned to standard chow for one week, and the binge episodes were repeated two more times following the same procedure. All food measurements were performed between 5:15-6:00 pm and 8:00-8:45 pm. This assay represents limited access to a highly palatable diet which results in binge-type eating behavior (Corwin, 2006). To measure non-binge behavior, the rats were given continuous access to the high-fat diet for one week. On day 7, food intake was again measured over two hours (6:00-8:00 pm) beginning at the onset of the dark cycle. Continuous access to the high-fat diet does not result in binge-type eating behavior.

\subsection{Fat preference}

The three diets used for the fat preference study $(10 \%, 12.5 \%$, and $20 \%$ fat by kcal) were made by Research Diets (Table 1) and milled on the same day to minimize variability. The $12.5 \%$ fat and $20 \%$ fat diets were based on the $10 \%$ fat diet (Research Diets OpenSource diet D12450B) and formulated to vary the fat content while maintaining the same ratio of 
sucrose to corn starch between diets. At the beginning of the experiment, two food hoppers containing $10 \%$ fat diet were placed into the rat cages for three days to allow the rats to habituate to minimize food neophobia. Then, for two days, rats were given ad libitum access to two diets in separate food hoppers, one containing $12.5 \%$ dietary fat and the other containing 20\% dietary fat. The weights of the hoppers were recorded daily between 2:004:00 pm and the positions of the hoppers were alternated each day to prevent a position bias. All food hoppers were washed and randomized.

\section{Results}

\subsection{Generation and validation of NMUR2 knockdown}

We created plasmids designed to knock down NMUR2 to investigate how NMUR2 signaling in the PVN contributes to the regulation of body weight and feeding behaviors. To achieve optimal knockdown efficiency, ten different shRNA sequences engineered to knock down NMUR2 were screened in vitro, and subsequent NMUR2 mRNA expression was quantified using real-time PCR. We found that transfection with two out of the ten hairpins (shNMUR2 \#5 and shNMUR2 \#9) resulted in approximately 95\% knockdown of NMUR2 mRNA in vitro, compared to a non-silencing control hairpin, shCTRL (Fig. 1A). In addition to packaging the shCTRL hairpin into AAV (AAV-shCTRL), we selected shNMUR2 \#5 to package into an AAV (AAV-shNMUR2) for in vivo testing because it achieved the greatest knock down of NMUR2 mRNA at 95.1\%. Furthermore, we evaluated shNMUR2 \#5 for offtarget activity by quantifying mRNA transcript levels of six of the closest matching mRNA sequences for knock down in cell culture. shNMUR2 \#5 did not significantly knock down the mRNA transcript levels of any of the sequences screened. Therefore, the observed in vivo effects are likely due to specific knock down of NMUR2 mRNA.

Viral knock down of NMUR2 in vivo was visualized and verified in ten rats (five for each virus) through double-label fluorescent immunostaining of NMUR2 and GFP in coronal sections containing PVN. The AAV vector employed contains a GFP tag, thus, neurons infected with the AAV viruses should express GFP. Indeed, GFP immunoreactivity (green) was readily detected in the PVN of shCTRL ${ }^{\mathrm{PVN}}$ and shNMUR2 ${ }^{\mathrm{PVN}}$ rats, suggesting a high level of transduction with either virus (Fig. 1C). As expected NMUR2 staining was abundant in the PVN of shCTRL ${ }^{\mathrm{PVN}}$ rats and was frequently colocalized with GFP expressing neurons in these animals (white arrows, Fig. 1C). Conversely, only a few NMUR2-labeled cells were detected in the shNMUR2 ${ }^{\mathrm{PVN}}$ rats, and we detected very little co-localization of GFP and NMUR2 in the PVN following AAV-shNMUR2 transduction. This suggests that the shNMUR2 virus successfully knocked down endogenous NMUR2 expression in infected neurons in vivo.

In vitro antibody validation confirmed the specificity of our rabbit polyclonal anti-NMUR2 primary antibody. Over-expression of NMUR2 in HEK-293 cells demonstrates bright and robust immunostaining for NMUR2. Non-transfected control cells showed light staining for NMUR2 because of low levels of endogenous protein expression (data not shown). Furthermore, NMUR2 immunostaining is highly enriched in the PVN, a pattern which closely matches the observed distribution of NMUR2 mRNA via in situ hybridization thus supporting the specificity of the antibody (Howard et al., 2000, Graham et al., 2003).

\subsection{Food intake on standard chow versus high-fat diet}

To test the hypothesis that knock down of NMUR2 in the PVN would increase food consumption, we evaluated food intake in shNMUR2 ${ }^{\mathrm{PVN}}$ and shCTRL ${ }^{\mathrm{PVN}}$ animals. Food intake was measured every afternoon for one week on a standard chow and one week on a high-fat diet (Fig. 2). We analyzed data collected on daily food intake with a repeated 
measures 2-way ANOVA, followed by a Bonferroni correction for multiple comparisons. When rats were fed a standard chow, no significant differences were observed between the groups' daily food intake (Fig. 2A). However, when animals were fed a high-fat diet, the shNMUR $2^{\mathrm{PVN}}$ rats ate significantly more than controls $(F(1,14)=9.628, p<0.01)$, and the post-hoc test showed significant differences between groups on day $4(p<0.05)$, day 6 $(p<0.05)$, and day 7 ( $p<0.01)$ (Fig. 2B). Two-way ANOVA analysis of cumulative food intake for each group on the two diets (expressed as average daily food intake) revealed that the shNMUR2 ${ }^{\mathrm{PVN}}$ rats ate significantly more food over the one week period than shCTRL $^{\text {PVN }}$ rats on a high-fat diet, but not on a standard chow (Fig. $2 \mathrm{C} ; \mathrm{F}(1,28)=8.96$, $p<0.01)$. To eliminate the possibility that differences in food intake were related to differences in locomotor activity, we placed the rats in locomotor chambers and measured their activity over 24 hours. No significant differences in locomotor activity were observed between groups during the dark or light cycle (Fig. 2D; 2-way ANOVA; F(1,29)=0.63, $p=0.43)$.

\subsection{Body weight and weight gain on standard chow versus high-fat diet}

To evaluate the effect of NMUR2 knockdown in the PVN on body weight and weight gain, we monitored body weight during the food intake studies. While being maintained on a standard chow, unpaired two-tailed t-tests revealed there were no significant differences in body weight (Fig. 3A; $\mathrm{t}=1.21 ; p=0.25$ ) or weight gain (Fig. 3B; $\mathrm{t}=0.029 ; p=0.40$ ) in the shNMUR2 ${ }^{\text {PVN }}$ rats compared to the shCTRL ${ }^{\text {PVN }}$ rats, with average body weights of $436.0 \mathrm{~g}$ vs. $417.7 \mathrm{~g}$ and average weight gain of $11.3 \mathrm{~g}$ and $11.4 \mathrm{~g}$, respectively. However, when placed on a $45 \%$ high-fat diet, the shNMUR2 ${ }^{\mathrm{PVN}}$ rats had significantly increased body weights (Fig. 3C; $\mathrm{t}=1.96 ; p<0.05$ ) due to significantly greater weight gain (Fig. 3D; $\mathrm{t}=3.49 ; p<0.01$ ) compared to control. Specifically, the average body weight of the shNMUR2 ${ }^{\text {PVN }}$ rats was 478.6g and that of shCTRL PVN rats was $447.6 \mathrm{~g}$, with an average weight gain over the seven day period of $42.6 \mathrm{~g}$ and $29.9 \mathrm{~g}$, respectively. On a high-fat diet, shNMUR2 ${ }^{\mathrm{PVN}}$ rats demonstrated a significantly higher feed efficiency than the shCTRL ${ }^{\text {PVN }}$ rats (Fig. 3E; $\mathrm{t}=3.02 ; p<0.05)$. Feed efficiency was calculated by dividing the weight gain in grams by the feed consumption (kcal) and suggests possible metabolic effects of NMUR2 knockdown in the PVN.

\subsection{Binge-type eating}

We hypothesized that NMUR2 knockdown would enhance binge-type eating relative to controls. Binge-type eating was assessed by measuring the intake of $45 \%$ high-fat diet during the first two hours of the dark cycle, after the rats had been maintained on a standard chow for seven days. To demonstrate that this limited-access approach results in binge-type eating, we first monitored the animals' intake of a standard chow during the two hour period on the day before the binge began, similar to the time frame when the rats would be bingeing on the high-fat diet. We observed a normal intake of standard chow and no significant difference between shCTRL ${ }^{\mathrm{PVN}}$ and shNMUR2 ${ }^{\mathrm{PVN}}$ rats (Fig. 4A). Next we quantified non-binge intake of the $45 \%$ high-fat diet by giving the animals continuous access to the food for seven days and measured two hour intake on day seven (Fig. 4B). This continuous access did not result in significant differences between the groups and established the baseline intake used to interpret the binge-type eating. For the binge-type eating assay (Fig. 4C), as expected both groups of animals binged on the $45 \%$ high-fat diet by increasing their food intake over baseline non-binge intake during the two hour period, but the binge intake of shNMUR2 ${ }^{\mathrm{PVN}}$ rats $(9.38 \mathrm{~g})$ was significantly greater than the binge intake of shCTRL ${ }^{\text {PVN }}$ rats $(7.39 \mathrm{~g})$ (Fig. $4 \mathrm{C}$; $\mathrm{t}$-test; $\left.\mathrm{t}=2.08 ; p<0.05\right)$. 


\subsection{Sucrose preference and fat preference}

Preference for solutions containing $0.25 \%, 0.5 \%$, or $1.0 \%$ sucrose was determined by placing two water bottles in the cage, giving the rats a choice between drinking from a bottle containing one of these sucrose solutions or water. Low sucrose concentrations were chosen based on previous publications (Bolanos et al., 2003, Green et al., 2008) and experience (Ignar et al., 2011). Furthermore, the sensitivity threshold of rats for sucrose is $0.09 \%$ (Sclafani and Nissenbaum, 1987); these concentrations provide a more sensitive detection of differences in sucrose preference. Rats were exposed to two bottles at a time, one containing water, and one containing a given concentration of sucrose for a total of 48 hours per pairing. Intake was measured by weighing the bottles, and percent preference was calculated by taking the two-day total sucrose intake and dividing it by the total amount consumed from both the sucrose and water bottle. No significant differences in consumption were observed between the shNMUR2 ${ }^{\mathrm{PVN}}$ rats versus the shCTRL ${ }^{\mathrm{PVN}}$ rats at any concentration of sucrose based on a two-way ANOVA (Fig. 5A; $\mathrm{F}(1,42)=0.035 ; p=0.85$ ), suggesting that NMU regulation of feeding behavior is specific to dietary fat versus carbohydrates.

To determine fat preference, two food hoppers were placed in the cages, giving the rats a choice between diets containing $12.5 \%$ or $20 \%$ fat by calories. Preference for the $20 \%$ fat diet was calculated by taking the two-day total food intake of each diet and dividing it by the total amount consumed across both diets and therefore represents the percent of the animal's diet that was comprised of higher-fat food. We controlled for total food consumption by dividing the grams consumed of the $20 \%$ fat diet by the total food consumption (grams of $20 \%$ fat diet plus grams of $12.5 \%$ fat diet). After having ad libitum access to the diets for 48 hours, the shNMUR2 ${ }^{\mathrm{PVN}}$ rats had an average preference score of 0.84 for the $20 \%$ fat diet; this was significantly higher than shCTRL ${ }^{\mathrm{PVN}}$ rats which had an average preference score of 0.64 (Fig. 5B; t-test; $\mathrm{t}=2.29 ; p<0.05$ ). Furthermore, the shNMUR2 ${ }^{\mathrm{PVN}}$ rats consumed significantly more of the higher-fat diet than the controls (Fig. 5C; F $(1,28)=1.20 ; 2$-way ANOVA; $p<0.01)$. These data demonstrate that shNMUR2 ${ }^{\mathrm{PVN}}$ rats prefer a higher-fat diet more than shCTRL ${ }^{\mathrm{PVN}}$ rats.

\section{Discussion}

Based upon data indicating that some NMUR2-null mice have increased bodyweight on a high-fat diet relative to controls (Egecioglu et al., 2009), and data indicating that infusion of NMU directly into the PVN suppresses food intake (Wren et al., 2002), we hypothesized that NMUR2 signaling in the PVN specifically modifies intake of a high-fat diet. We tested this hypothesis by knocking down NMUR2 specifically in the PVN of adult rats followed by measuring food intake, body weight, food preference, binge-type eating, and locomotor activity. Four principal findings emerged:

First, NMUR2 knockdown increased intake of a high-fat diet. This demonstrates that NMUR2 signaling in the PVN influences intake of obesogenic food. Conversely, we did not observe significant differences in intake of a standard chow, implying that NMUR2 signaling is specific to the high-fat diet.

Second, shNMUR2 ${ }^{\mathrm{PVN}}$ rats had higher body weights when given ad libitum access to this high-fat diet, with no effect on body weight relative to controls when maintained on a standard chow. This indicates that endogenous NMUR2 expression in the PVN minimizes weight gain in an obesogenic environment. That is, NMUR2 signaling in the PVN normally blunts weight gain even when exposed to obesogenic food. Therefore, when NMUR2 expression was knocked down, weight gain increased. 
Third, shNMUR2 $2^{\mathrm{PVN}}$ rats have an increased preference for dietary fat, but not for sucrose. Fat and sugar are both reinforcing components of food. However, in rodents, canines, and non-human primates, dietary fat is more effective at inducing obesity than carbohydrates (Warwick and Schiffman, 1992, West and York, 1998). For example, the high-fat diet used in our studies has $45 \%$ of the calories from fat and $30 \%$ of the calories from carbohydrates (Van Heek et al., 1997). This diet effectively increases adiposity and is therefore considered to be obesogenic. Non-obesogenic diets typically contain around $15 \%$ of the calories from fat, and can contain as much as $70 \%$ of the calories from carbohydrates. Although some receptors (e.g. mu-opioid receptor) regulate consumption of carbohydrates and sugars (Ignar et al., 2011), our data suggest that NMUR2 signaling in the PVN selectively regulates preference for increased dietary fat.

Fourth, NMUR2 knockdown in the PVN increases binge-type eating behavior relative to controls. Although the shNMUR2 ${ }^{\mathrm{PVN}}$ rats demonstrate increased daily intake of an obesogenic diet, binge eating of obesogenic food is thought to be driven by distinct neurocircuitry. For example, dopamine is released into the nucleus accumbens during repeated binge eating episodes, which does not occur in non-bingeing rats (Corwin et al., 2011). Interestingly, the present study indicates that NMUR2 signaling in the PVN inhibits food intake during binge-type episodes, in addition to generally suppressing consumption of an obesogenic diet.

An outstanding candidate for the source of NMU is the arcuate nucleus. NMU is highly expressed in this region of the brain (Howard et al., 2000, Graham et al., 2003), and the arcuate nucleus projects directly to the PVN (Schwartz et al., 2000). This could be somewhat comparable to the melanocortin system which arises in the arcuate nucleus and signals to melanocortin-4 receptors in the PVN. This system is well established as an important regulator of food intake and body weight (Schwartz et al., 2000). However, the relationship between NMU and the melanocortin systems is unclear. For example, are NMU and a-melanocyte-stimulating hormones expressed in the same neurons in the arcuate nucleus? Also, are NMUR2 and melanocortin-4 receptors expressed in the same neurons in the PVN?

The neuroanatomical target of NMUR2-containing neurons in the PVN could be the parabrachial nucleus. The parabrachial nucleus is capable of modulating food preference and feeding behavior via regulation of dopamine release in the limbic system (Norgren et al., 2006). Although the parabrachial nucleus primarily receives projections from second-order taste neurons found in the nucleus of the solitary tract, it also receives afferents directly from the PVN (Kirchgessner et al., 1988). Therefore, NMUR2 neurons may project to the parabrachial nucleus which would in turn modify dopamine release in regions of the limbic system such as the nucleus accumbens and ultimately alter feeding behaviors specific to higher-fat foods.

Taken together, these data demonstrate that decreased NMUR2 signaling in the PVN of adult rats promotes consumption of high-fat (obesogenic) food and increased body weight. This poses the idea that dysregulation of NMU signaling may promote obesity due to increased consumption of obesogenic food. Consistent with this concept, variations in the NMU amino acid sequence are associated with obesity in humans (Hainerova et al., 2006) suggesting that NMU-NMUR2 signaling is translationally relevant. Therefore, NMUR2 agonism represents a promising therapeutic strategy for treating obesity. 


\section{Acknowledgments}

We would like to thank James Kasper for reading and revising the manuscript. These studies were conducted with the support of the National Institute of Health: UL1TR000071 (NCATS), P30DK079638 (NIDDK), P30DA028821 (NIDA), KL2TR000072 (NCATS), and T32DA07287 (NIDA).

\section{List of Abbreviations}

$\begin{array}{ll}\text { NMU } & \text { Neuromedin U } \\ \text { NMUR2 } & \text { Neuromedin U receptor 2 } \\ \text { PVN } & \text { Paraventricular nucleus } \\ \text { AAV } & \text { Adeno-associated virus } \\ \text { ShRNA } & \text { small hairpin RNA } \\ \text { GFP } & \text { green fluorescent protein } \\ \text { ShCTRL } & \text { small hairpin control (non-silencing) RNA } \\ \text { ShNMUR2 } & \text { small hairpin NMUR2 knockdown RNA } \\ \text { DAPI } & \text { 4',6-diamidino-2-phenylindole }\end{array}$

\section{References}

Bolanos CA, Barrot M, Berton O, Wallace-Black D, Nestler EJ. Methylphenidate treatment during pre- and periadolescence alters behavioral responses to emotional stimuli at adulthood. Biol Psychiatry. 2003; 54:1317-1329. [PubMed: 14675795]

Brighton PJ, Szekeres PG, Willars GB. Neuromedin U and its receptors: structure, function, and physiological roles. Pharmacol Rev. 2004; 56:231-248. [PubMed: 15169928]

Corwin RL. Bingeing rats: a model of intermittent excessive behavior? Appetite. 2006; 46:11-15. [PubMed: 16188345]

Corwin RL, Avena NM, Boggiano MM. Feeding and reward: perspectives from three rat models of binge eating. Physiol Behav. 2011; 104:87-97. [PubMed: 21549136]

Egecioglu E, Ploj K, Xu X, Bjursell M, Salome N, Andersson N, Ohlsson C, Taube M, Hansson C, Bohlooly YM, Morgan DG, Dickson SL. Central NMU signaling in body weight and energy balance regulation: evidence from NMUR2 deletion and chronic central NMU treatment in mice. American journal of physiology Endocrinology and metabolism. 2009; 297:E708-716. [PubMed: 19584200]

Finucane MM, Stevens GA, Cowan MJ, Danaei G, Lin JK, Paciorek CJ, Singh GM, Gutierrez HR, Lu Y, Bahalim AN, Farzadfar F, Riley LM, Ezzati M. National, regional, and global trends in bodymass index since 1980: systematic analysis of health examination surveys and epidemiological studies with 960 country-years and 9.1 million participants. Lancet. 2011; 377:557-567. [PubMed: 21295846]

Graham ES, Turnbull Y, Fotheringham P, Nilaweera K, Mercer JG, Morgan PJ, Barrett P. Neuromedin $\mathrm{U}$ and Neuromedin $\mathrm{U}$ receptor-2 expression in the mouse and rat hypothalamus: effects of nutritional status. J Neurochem. 2003; 87:1165-1173. [PubMed: 14622096]

Green TA, Alibhai IN, Unterberg S, Neve RL, Ghose S, Tamminga CA, Nestler EJ. Induction of activating transcription factors (ATFs) ATF2, ATF3, and ATF4 in the nucleus accumbens and their regulation of emotional behavior. J Neurosci. 2008; 28:2025-2032. [PubMed: 18305237]

Guh DP, Zhang W, Bansback N, Amarsi Z, Birmingham CL, Anis AH. The incidence of comorbidities related to obesity and overweight: a systematic review and meta-analysis. BMC Public Health. 2009; 9:88. [PubMed: 19320986]

Hainerova I, Torekov SS, Ek J, Finkova M, Borch-Johnsen K, Jorgensen T, Madsen OD, Lebl J, Hansen T, Pedersen O. Association between neuromedin U gene variants and overweight and obesity. J Clin Endocrinol Metab. 2006; 91:5057-5063. [PubMed: 16984985] 
Hanada R, Teranishi H, Pearson JT, Kurokawa M, Hosoda H, Fukushima N, Fukue Y, Serino R, Fujihara H, Ueta Y, Ikawa M, Okabe M, Murakami N, Shirai M, Yoshimatsu H, Kangawa K, Kojima M. Neuromedin U has a novel anorexigenic effect independent of the leptin signaling pathway. Nat Med. 2004; 10:1067-1073. [PubMed: 15448684]

Hommel JD, Sears RM, Georgescu D, Simmons DL, DiLeone RJ. Local gene knockdown in the brain using viral-mediated RNA interference. Nat Med. 2003; 9:1539-1544. [PubMed: 14634645]

Hommel JD, Trinko R, Sears RM, Georgescu D, Liu ZW, Gao XB, Thurmon JJ, Marinelli M, DiLeone RJ. Leptin receptor signaling in midbrain dopamine neurons regulates feeding. Neuron. 2006; 51:801-810. [PubMed: 16982424]

Howard AD, Wang R, Pong SS, Mellin TN, Strack A, Guan XM, Zeng Z, Williams DL Jr, Feighner SD, Nunes CN, Murphy B, Stair JN, Yu H, Jiang Q, Clements MK, Tan CP, McKee KK, Hreniuk DL, McDonald TP, Lynch KR, Evans JF, Austin CP, Caskey CT, Van der Ploeg LH, Liu Q. Identification of receptors for neuromedin $U$ and its role in feeding. Nature. 2000; 406:70-74. [PubMed: 10894543]

Ignar DM, Goetz AS, Noble KN, Carballo LH, Stroup AE, Fisher JC, Boucheron JA, Brainard TA, Larkin AL, Epperly AH, Shearer TW, Sorensen SD, Speake JD, Hommel JD. Regulation of Ingestive Behaviors in the Rat by GSK1521498, a Novel \{micro\}-Opioid Receptor-Selective Inverse Agonist. J Pharmacol Exp Ther. 2011; 339:24-34. [PubMed: 21712426]

Institute of Laboratory Animal Resources (U.S.). Guide for the care and use of laboratory animals. Washington, D.C: National Academy Press; 1996.

Kirchgessner AL, Sclafani A, Nilaver G. Histochemical identification of a PVN-hindbrain feeding pathway. Physiol Behav. 1988; 42:529-543. [PubMed: 2842813]

Norgren R, Hajnal A, Mungarndee SS. Gustatory reward and the nucleus accumbens. Physiol Behav. 2006; 89:531-535. [PubMed: 16822531]

Ogden, CL.; Carroll, MD.; Kit, BK.; Flegal, KM. NCHS Data Brief. 2012. Prevalence of Obesity in the United States, 2009-2010; p. 82

Paxinos, G.; Watson, C. The rat brain in stereotaxic coordinates. Amsterdam; Boston: Elsevier Academic Press; 2005.

Peier A, Kosinski J, Cox-York K, Qian Y, Desai K, Feng Y, Trivedi P, Hastings N, Marsh DJ. The antiobesity effects of centrally administered neuromedin $\mathrm{U}$ and neuromedin $\mathrm{S}$ are mediated predominantly by the neuromedin U receptor 2 (NMUR2). Endocrinology. 2009; 150:3101-3109. [PubMed: 19324999]

Schwartz MW, Woods SC, Porte D Jr, Seeley RJ, Baskin DG. Central nervous system control of food intake. Nature. 2000; 404:661-671. [PubMed: 10766253]

Sclafani A, Nissenbaum JW. Taste preference thresholds for Polycose, maltose, and sucrose in rats. Neurosci Biobehav Rev. 1987; 11:181-185. [PubMed: 3614784]

Van Heek M, Compton DS, France CF, Tedesco RP, Fawzi AB, Graziano MP, Sybertz EJ, Strader CD, Davis HR Jr. Diet-induced obese mice develop peripheral, but not central, resistance to leptin. J Clin Invest. 1997; 99:385-390. [PubMed: 9022070]

Warwick ZS, Schiffman SS. Role of dietary fat in calorie intake and weight gain. Neurosci Biobehav Rev. 1992; 16:585-596. [PubMed: 1480353]

West DB, York B. Dietary fat, genetic predisposition, and obesity: lessons from animal models. Am J Clin Nutr. 1998; 67:505S-512S. [PubMed: 9497161]

Wren AM, Small CJ, Abbott CR, Jethwa PH, Kennedy AR, Murphy KG, Stanley SA, Zollner AN, Ghatei MA, Bloom SR. Hypothalamic actions of neuromedin U. Endocrinology. 2002; 143:42274234. [PubMed: 12399416] 
- Knock down of NMUR2 in the PVN stimulates intake of an obesogenic diet resulting in increased body weight

- NMUR2 signaling in the PVN regulates dietary preference

- NMUR2 knockdown in the PVN increases binge-type eating behavior 
A

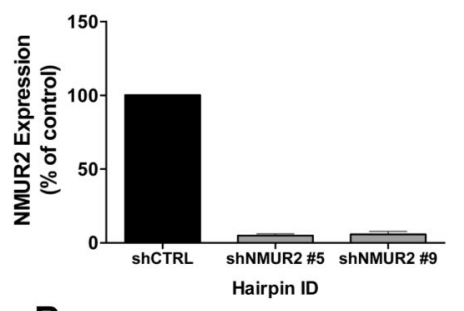

B
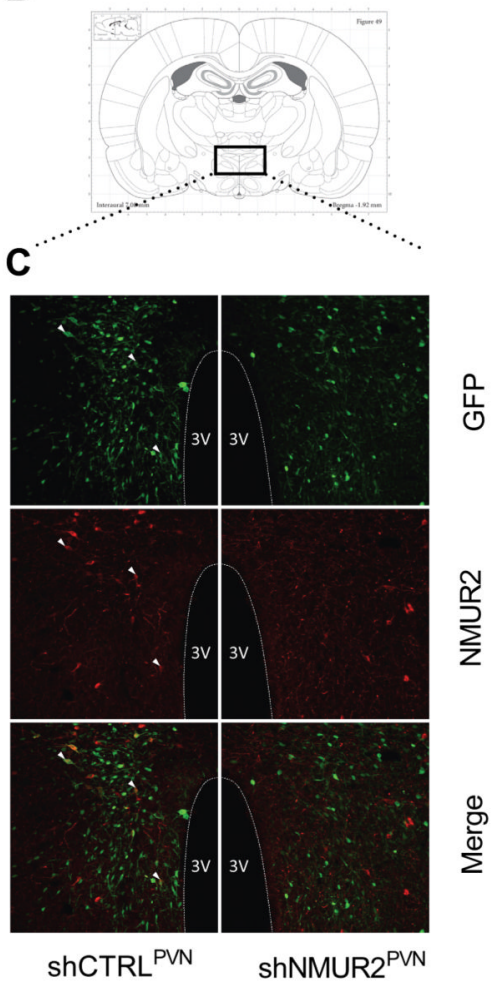

Figure 1.

shRNA-mediated knockdown of NMUR2 in vitro and in vivo. $\boldsymbol{A}$, Screening of hairpins designed to knock down NMUR2. Transient transfection of hairpins designed to knock down NMUR2 (shNMUR2) in HEK293 cells results in approximately 95\% knockdown verses shCTRL (shNMUR2 \#5 and shNMUR2 \#9). B, Atlas plate showing approximate location of immunohistochemical images. $\boldsymbol{C}$, Double label fluorescent immunostaining of NMUR2 (red) or GFP (green, marker of transduction) in the PVN of adult rats.

Colocalization between NMUR2 and GFP is readily observed in neurons infected with shCTRL virus (three of the co-localized neurons are marked by white arrows, left panels) while colocalization is only rarely observed in neurons infected with shNMUR2 virus (right panels). 
A
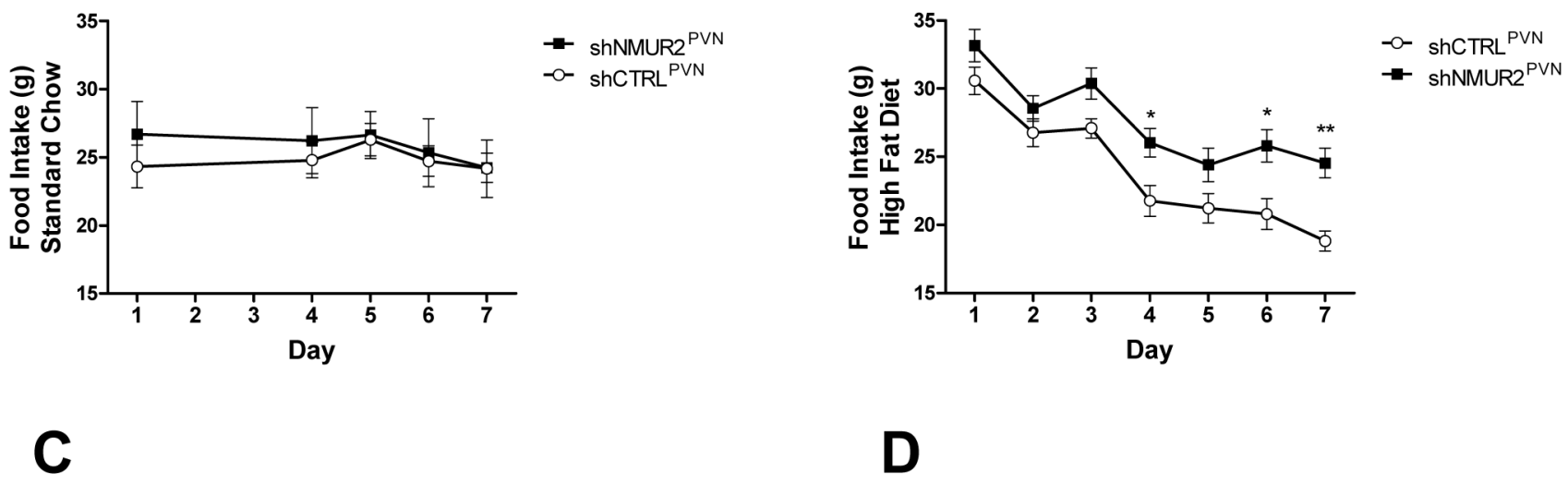

B

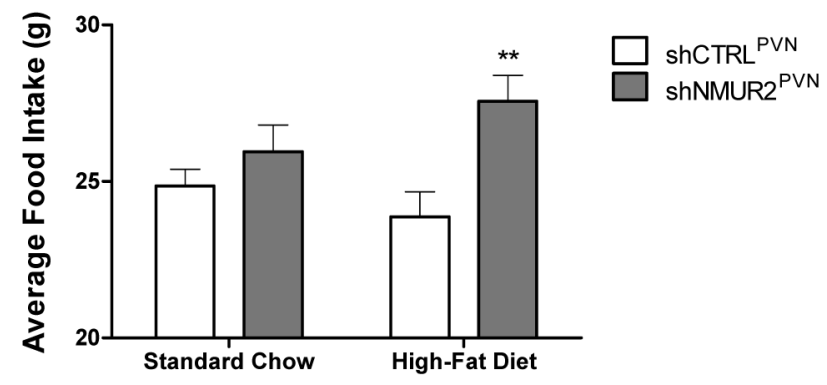

D

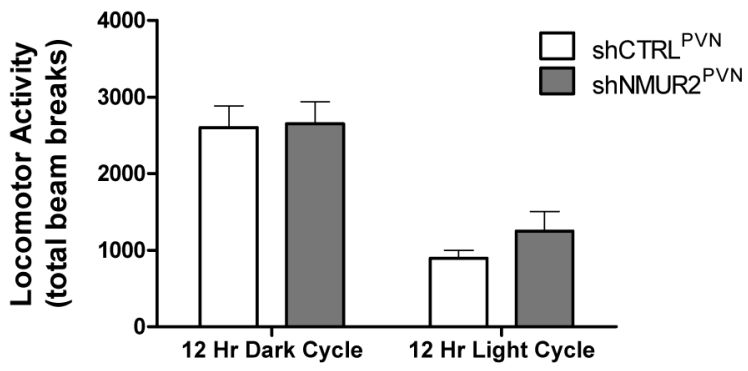

Figure 2.

Knock down of NMUR2 in the PVN increases intake of a high-fat diet but not a standard chow. Daily food intake over seven days on a standard chow $(\boldsymbol{A})$ or on a high-fat diet $(\boldsymbol{B})$ in rats receiving the shNMUR2 virus $(\mathrm{n}=9$; closed squares) verses the shCTRL virus $(\mathrm{n}=7$; open circles). $\boldsymbol{C}$, Average daily food intake is significantly elevated in shNMUR2 rats (gray bars) verses shCTRL rats (white bars) on a high-fat diet, but not on a standard chow. $\boldsymbol{D}$, Home cage locomotor activity (expressed as total beam breaks) is not different during the light or dark cycle in shNMUR 2 verses shCTRL rats. $(* \mathrm{p}<0.05$, ** $\mathrm{p}<0.01$ by ANOVA followed post hoc test). Error bars represent the standard error of the mean. 

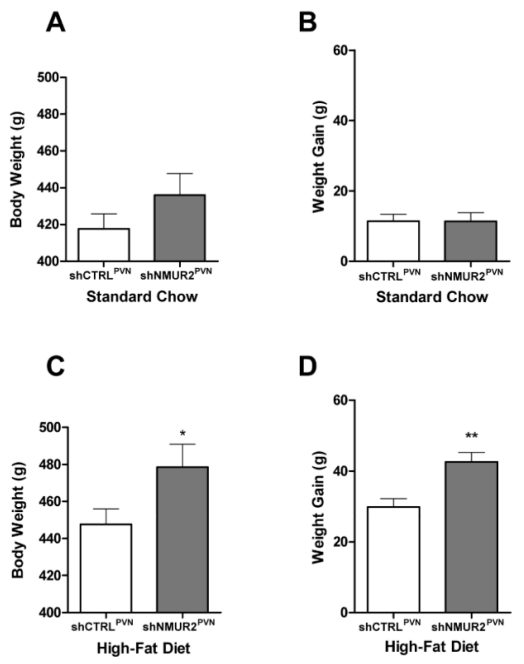

E

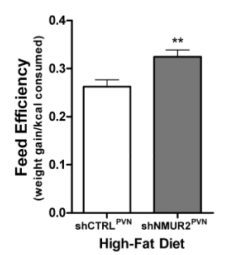

Figure 3.

NMUR2 knockdown in the PVN results in increased body weight, weight gain, and feed efficiency on a high-fat diet, but not on a standard chow. On a standard chow, shNMUR2 ${ }^{\text {PVN }}$ rats ( $\mathrm{n}=9$; gray bars) did not have altered total body weight $(\boldsymbol{A})$ or weight gain $(\boldsymbol{B})$ over seven days when compared to shCTRL ${ }^{\mathrm{PVN}}$ rats $(\mathrm{n}=7$; white bars). On a highfat diet, shNMUR2 $2^{\mathrm{PVN}}$ rats had significantly greater body weight $(\boldsymbol{C})$ and weight gain $(\boldsymbol{D})$ verses shCTRL ${ }^{P V N}$ rats. $\boldsymbol{E}$, The feed efficiency on a high-fat diet is enhanced by knock down of NMUR2. Feed efficiency is calculated as weight gain in grams divided by food consumption in kilocalories. $(* \mathrm{p}<0.05, * * \mathrm{p}<0.01$ by two-tailed $\mathrm{t}$-test). Error bars represent the standard error of the mean. 
A

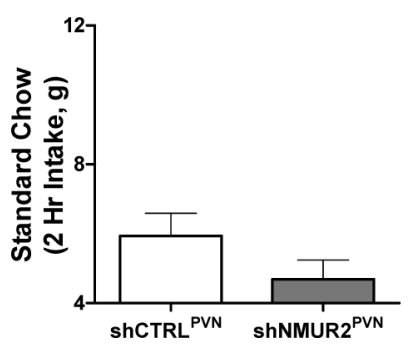

B

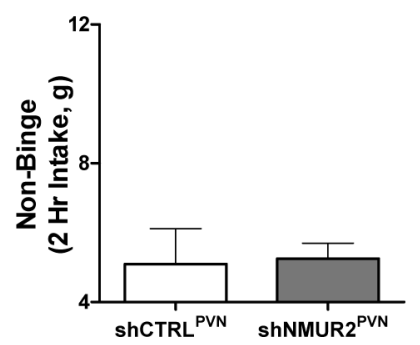

C

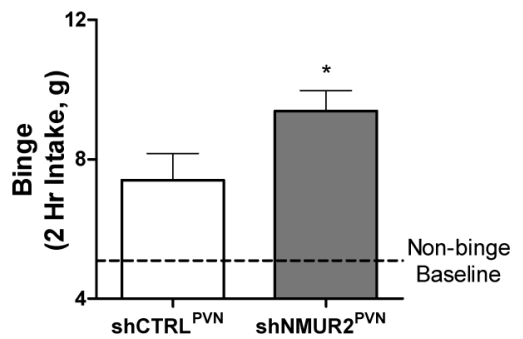

Figure 4.

Knock down of NMUR2 in the PVN modifies binge-type eating behavior. $\boldsymbol{A}$, Control assay demonstrating baseline intake of a standard chow over a 2 hour period. No significant differences were observed between groups. $\boldsymbol{B}$, Continuous access (non-binge) intake of a high-fat diet in shCTRL ${ }^{\mathrm{PVN}}$ and shNMUR2 ${ }^{\mathrm{PVN}}$ rats over a two hour period beginning at the onset of the dark cycle. No significant differences were observed between groups. $\boldsymbol{C}$, Two hour binge-type intake of a high-fat diet. NMUR2 knockdown ( $\mathrm{n}=9$; gray bars) potentiates binge-type eating in comparison to controls ( $n=7$; white bars) in animals receiving two hour access to a high-fat diet once per week. Dotted line represents average non-binge intake of a high-fat diet by control animals over two hours as demonstrated in panel (B). (* p $₫ 0.05$ by one-tailed t-test). Error bars represent the standard error of the mean. 
A

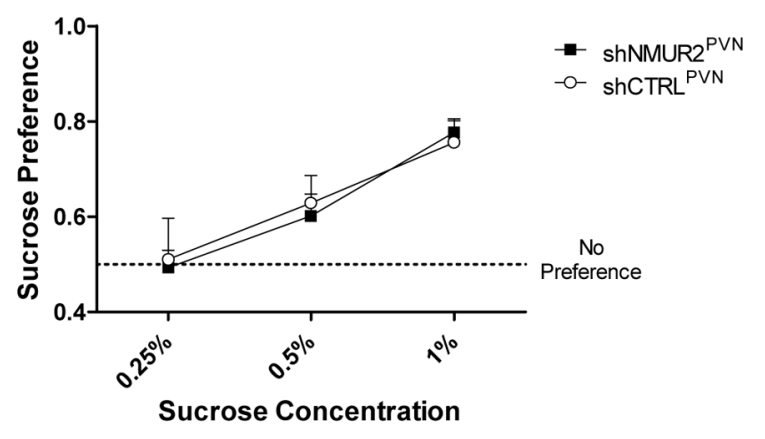

Sucrose Concentration
B

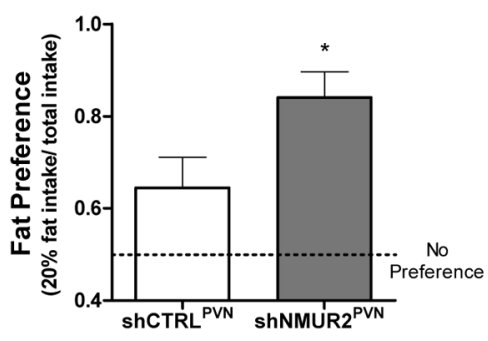

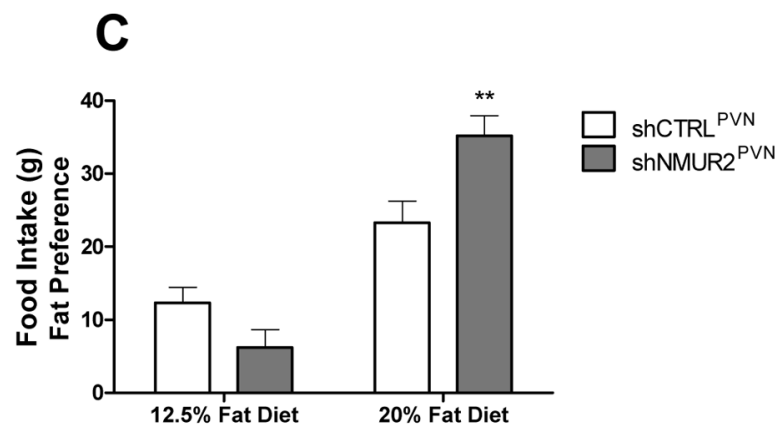

Figure 5.

Knock down of NMUR2 in the PVN enhances preference for dietary fat but not sucrose. $\boldsymbol{A}$, Preference for solutions containing $0.25 \%, 0.5 \%$, or $1.0 \%$ sucrose $(\mathrm{w} / \mathrm{v})$ relative to water alone. shNMUR2 ${ }^{\mathrm{PVN}}$ rats were not significantly different from shCTRL ${ }^{\mathrm{PVN}}$ rats at any concentration. $\boldsymbol{B}$, Preference score for diet containing $20 \%$ fat by calories verses diet containing $12.5 \%$ fat by calories when both diets are presented simultaneously. Over a 48 hour period, shNMUR2 ${ }^{\mathrm{PVN}}$ rats ( $\mathrm{n}=9$; gray bars) had a stronger preference for the higher-fat diet than controls ( $\mathrm{n}=7$; white bars) $(* \mathrm{p}<0.05$ by one-tailed t-test). $\boldsymbol{C}$, The total intake of the $20 \%$ fat diet and the $12.5 \%$ fat diet during the fat preference assay. shNMUR2 ${ }^{\mathrm{PVN}}$ rats (gray bars) consumed significantly more of the $20 \%$ fat diet than controls (white bars) $(* * \mathrm{p}<0.01$ verses shCTRL ${ }^{\text {PVN }}$ intake of $20 \%$ diet by AVOVA followed by Bonferroni correction for multiple comparisons). Both groups of rats consumed significantly more of the $20 \%$ diet than the $12.5 \%$ diet, significance not denoted. All error bars represent the standard error of the mean. 
